\title{
Off-Axis lons Extraction Simulation in Tubular Electron String Ion Source (TESIS)
}

\author{
A. Yu. Boytsov ${ }^{1}$ and A.A. Bulychev ${ }^{2}$ \\ ${ }^{1}$ Veksler And Baldin Laboratory of High Energy Physics, Joint Institute for Nuclear Research, 141980 Dubna, \\ Moscow region, Russia \\ ${ }^{2}$ Independent Researcher, Moscow, Russia
}

\begin{abstract}
Tubular Electron String Ion Source (TESIS) - improved version of Electron String Ion Source (ESIS) aiming for nearly 200-fold increase in ion yield - is under development at JINR. TESIS advantages over ESIS are discussed and their characteristics are compared. Basic scheme of TESIS operation is presented. One of the crucial processes in TESIS - off-axis ion extraction - requires special configuration of output electrodes providing steep potential gradient. Numerical simulations of ion extraction trajectories for two different electrode configurations are compared and the optimal one is discussed. The obtained results will be useful for TESIS design and construction.
\end{abstract}

\section{Introduction}

ESIS has been developed in Joint Institute for Nuclear Research since 1994 [1]. As the underlying principle of operation it utilizes electron string phenomenon that occurs in reflex mode of Electron Beam Ion Source (EBIS) [2] (for this reason, further in the text electron beam in ESIS and TESIS is called electron string). Source of this type allows to produce pulses of highly charged ions, such as $\mathrm{Xe}^{44+}$ with $7 \mu$ s pulse duration and $1.5 \cdot 10^{7}$ ions per pulse [3]. Various applications - ion beam surface modification, cancer radiation therapy and high-energy physics - may benefit from increased amount of ions in the beam, therefore it is desirable to increase ion yield of the source.

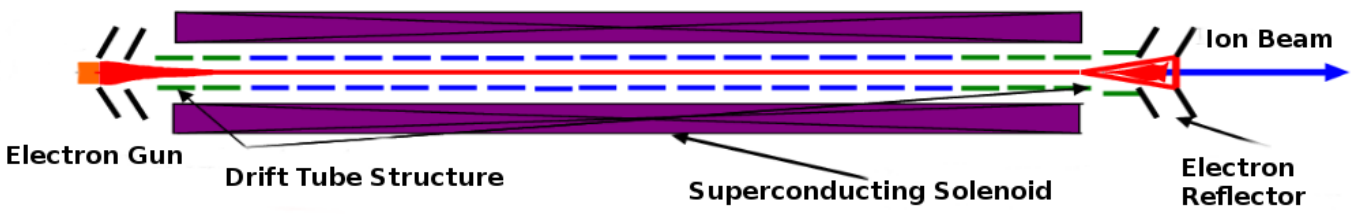

Figure 1. Electron String Ion Source scheme. Neutral atoms or molecules are injected into the drift tube where an electron string is formed and where they undergo electron-impact ionization. Combination of drift structure and electron string potentials allows to hold the ions long enough to achieve high degree of ionization. Typical length of the electron string is $1 \mathrm{~m}$ and string radius is $0.15 \mathrm{~mm}$ 


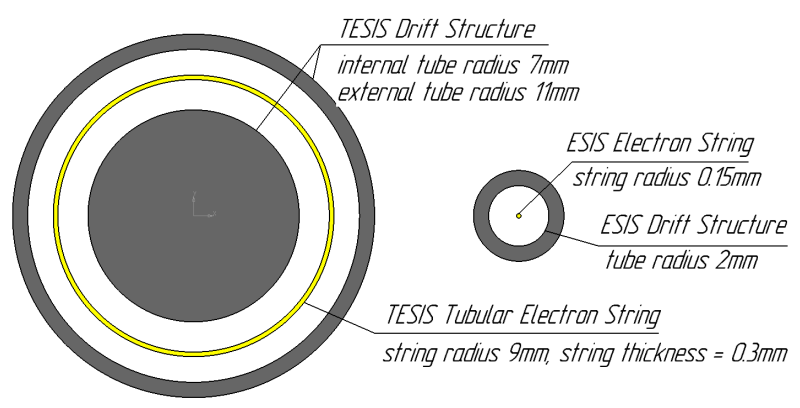

Figure 2. TESIS (left) and ESIS (right) drift structure cross sections. In TESIS, it is planned to form a tubular electron string with $1 \mathrm{~m}$ length, $9 \mathrm{~mm}$ internal radius and $0.3 \mathrm{~mm}$ thickness. Volume occupied by the string in TESIS almost 200 times larger than in ESIS, providing corresponding increase in ion yield.

Main parts of the ESIS are superconducting solenoid, electron gun, electron reflector and drift structure (see figure 1). Neutral atoms or molecules are injected into the drift structure where an electron string is formed and where they are ionized due to collisions with the electrons. Combination of electron string potential and carefully chosen voltages on the drift structure electrodes allows to confine ions inside the string long enough to achieve high degree of ionization. Ion yield depends on total charge of the electron string, which is limited by device parameters. Increase of the total electron string charge would allow to increase ion yield.

Comparison of drift structure cross sections for TESIS[4-6] and ESIS is shown on figure 2. In TESIS, it is planned to form a tubular electron string with $1 \mathrm{~m}$ length, $9 \mathrm{~mm}$ internal radius and $0.3 \mathrm{~mm}$ thickness. The volume occupied by the electrons would be almost 200 times larger compared to ESIS, providing corresponding increase in ion yield. Vacuum chamber and cryogenic system are ready for TESIS. Drift structure is under development.

Ion extraction procedure is crucial for ion source operation. Two major issues are beam focusing and ion extraction time. Regarding the focusing, in case of ESIS it is relatively easy to extract the ions along the central magnetic line. This is not possible in TESIS since magnetic field at the end of the solenoid is not strong enough to prevent divergence and focus tubular ion beam. To overcome this it was proposed to concentrate ions in a narrow area along few magnetic lines before extraction. Such solution would result in beam propagation direction nonparallel to the device axis, which should be taken into account by beam transport system. Besides, emittance of the beam would increase. If emittance gain would be too high for acceptance of the further device, it might be necessary to employ ion-ion cooling [7]. Regarding the extraction time, it should be less than $200 \mu$ s for practical applications. In order to fulfill both conditions, it is necessary to design a special geometry of the drift structure.

Preliminary calculations of ion extraction trajectories were made in [8]. However, to find the optimal configuration of extraction electrodes, more extensive simulation is required. Such modeling is concern of our present work. In the next section, details of the TESIS drift structure design are described, and in the section 3, results of numerical simulation of two particular configurations are presented.

\section{TESIS Drift Structure}

Conceptually, TESIS drift structure can be divided into three regions (see. figure 3). Neutral atoms are injected in the second one, where they are ionized and confined. After required ionization degree is 
reached, ions are extracted through the first region. In the third, potential barrier is formed to prevent the ions escape the trap.

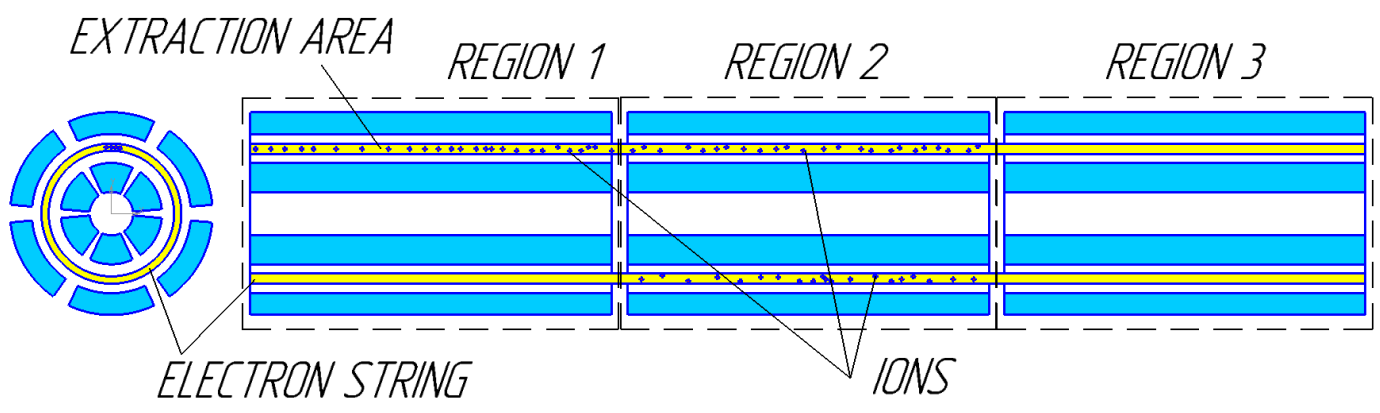

Figure 3. TESIS drift structure design. It can be divided into three regions: neutral atoms are injected and ionized in the second, then extracted through the first. In the third, potential barrier is formed to prevent ions escape the trap.

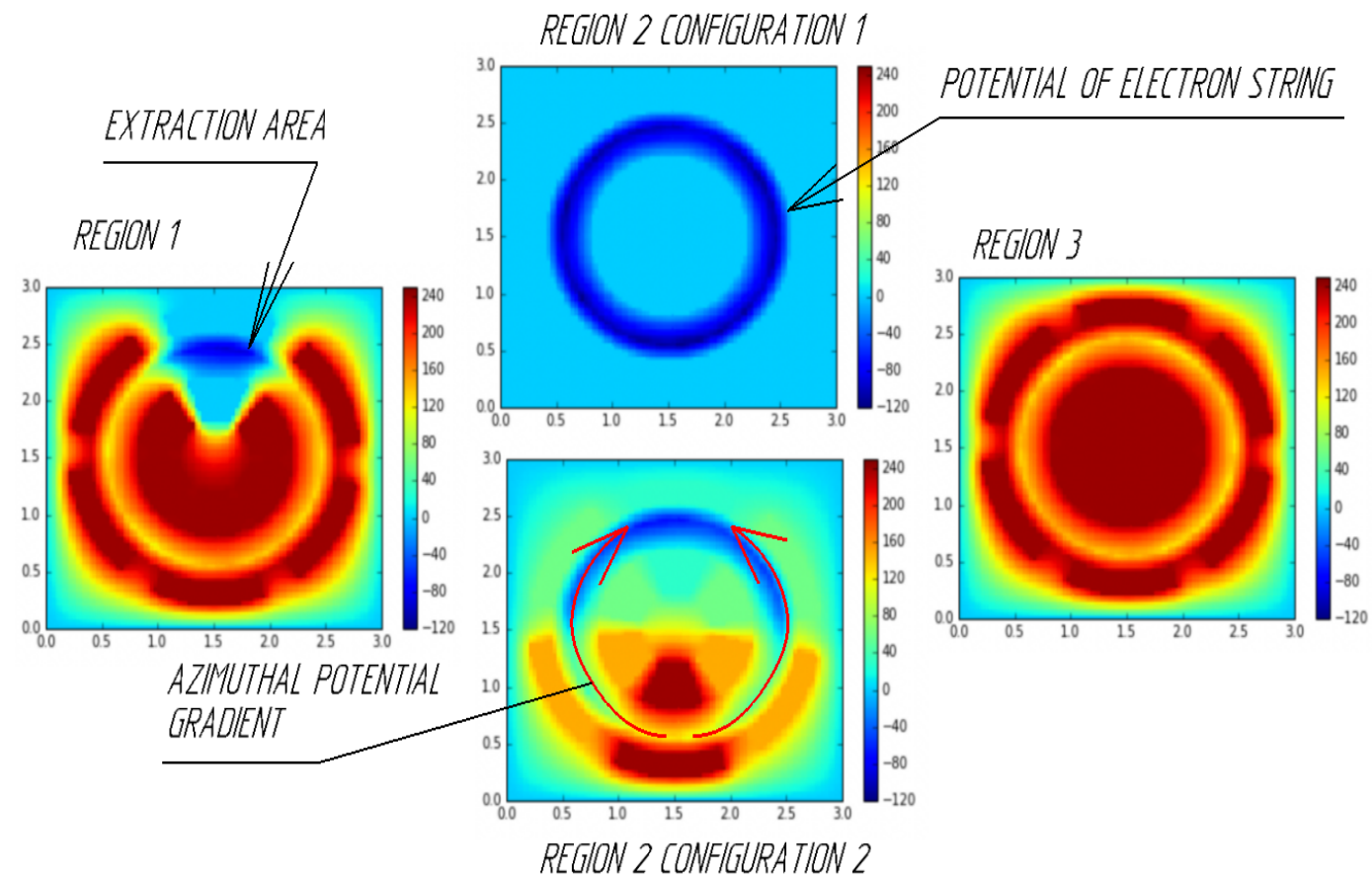

Figure 4. Potential in the drift structure. Two different configurations for the second region are compared. In the first one (top panel), ions drift along azimuthal angle in potential of the electron string. In the second one (bottom panel), voltages on the electrodes form azimuthal potential gradient to accelerate ions transition into extraction area of the first region.

In each region, there are electrodes arranged in circular fashion to form internal and external "tubes". Each "tube" is composed of six segments with gaps between them to achieve certain potential distribution. Electron string space-charge creates potential well in between the internal and external 

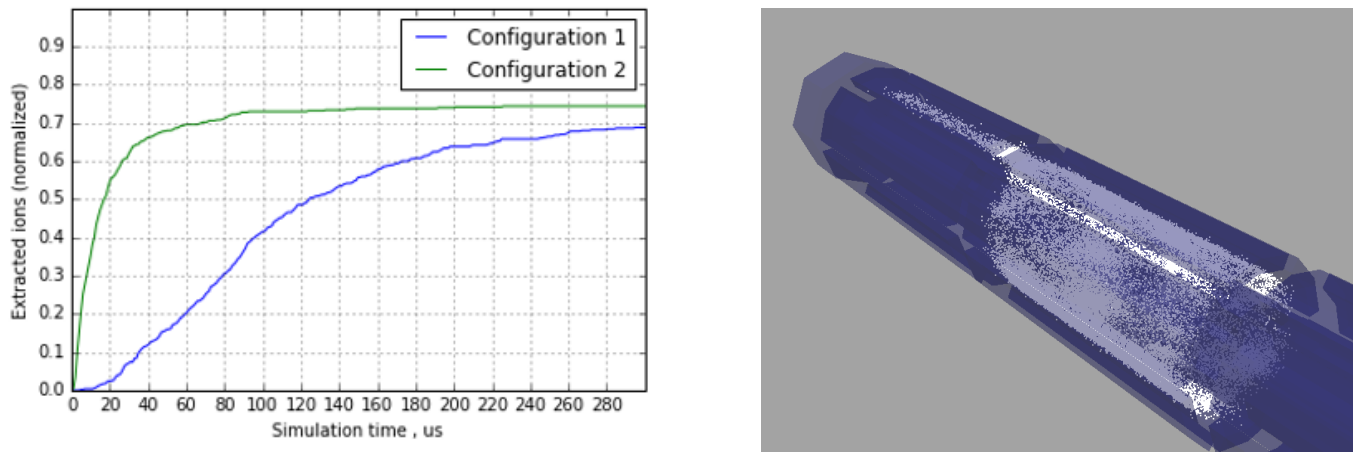

Figure 5. Left panel: total amount of ions extracted from the device relative to initial amount. Azimuthal potential gradient is absent in Configuration 1 and present in Configuration 2. The gradient significantly speedsup ion extraction. Right panel: ion distribution in TESIS during extraction. Ion beam in the extraction area is visible in the upper-left corner and the ion trap region in the bottom-right.

"tubes" of electrodes that confines ions in radial direction, whereas potential on the "tubes" confines them in the longitudinal direction.

In the first region there are five pairs of internal and external segments with constant barrier potential on them and a single pair with zero potential. The ions are extracted between the latter pair (see. figure 4).

In the third region, potentials forms a barrier, which reverts back the ions escaping the trap.

For the second region, two different configurations are considered. In order to form an output beam, it is necessary to collect the trapped ions near extraction area of the first region. In the first case (configuration 1), we do not take any specific measures to accelerate ion extraction. The ions drift along azimuthal angle inside radial potential well until they reach extraction area of the first region. In the second case (configuration 2), the voltages on the tube segments are chosen in such a way that an azimuthal potential gradient is created. It can accelerate ions transition into extraction area. It is important to understand the effect of azimuthal gradient on speed-up of ions extraction. If it is not significant, design of the drift structure can be simplified.

\section{Simulation}

To simulate ion extraction, Ef software [9] is used. It implements 3d electrostatic "particle-in-cell" [10] algorithm, and it also allows to calculate trajectories of charged particles under action of external electromagnetic fields neglecting particle-particle interaction.

Actual length of the drift structure is approximately $1 \mathrm{~m}$, divided into 23 sections of electrodes. Modeling the full length would require large amount of time and computational resources. To make it reasonable, only three sets of electrodes are used in simulation (see figure 3 ).

Simulation volume is $3 \times 3 \times 20 \mathrm{~cm}$ with $150 \times 150 \times 500$ rectilinear grid for fields computation. A total of 36 tube segments are placed in the volume. Their sizes are the same as on the figure 2; for beam dynamics internal tube outer radius $(7 \mathrm{~mm})$ and external tube inner radius $(11 \mathrm{~mm})$ are most relevant. Length of each segment is $5 \mathrm{~cm}$ with $1 \mathrm{~mm}$ gap between them.

To simulate ion extraction, 5000 macroparticles with proton charge-to-mass ratio are uniformly deposited in the area of electron string in the ion trap region (region 2 on figure 3). Trajectories of 
individual electrons are not simulated. Instead, since the electron string is largely unaffected by ions, the potential it creates was precomputed (on a similar grid) and loaded into ion simulation.

Longitudinal uniform magnetic field from the solenoid with $4 \mathrm{~T}$ amplitude is included in the simulation. Simulation time is $300 \mu$ s with $10^{-11} \mathrm{~s}$ step. Boris time integration scheme is used [10].

In both configurations it is necessary to set voltages on electrodes that would allow to form and extract ion beam. Barrier voltages should be high enough to keep the ions in the trap region. Besides, it is necessary to ensure that extraction efficiency is acceptable.

In both cases it was possible to achieve beam formation and extract about $70 \%$ of the ions in $200 \mu \mathrm{s}$ (see figure 5). The configuration with azimuthal potential gradient allows to significantly speed-up the process: at $10 \mu \mathrm{s} 30 \%$ of the ions are already extracted, whereas only about $5 \%$ in the other case. This amounts to 6 times difference in intensity. For example, ESIS is capable to deliver $3 \mathrm{nC}$ in $8 \mu \mathrm{s}$ ( $8 \mu \mathrm{s}$ pulses are required by JINR Nuclotron accelerator). Taking total amount of ions in the TESIS trap 200 larger than ESIS and ESIS extraction efficiency close to $100 \%$, we can somewhat optimistically hope for $180 \mathrm{nC}$ in configuration with the gradient and $30 \mathrm{nC}$ without it. Of course, these are crude estimates and in reality intensity would be smaller.

The electrode potentials in both configurations were selected empirically. It should be possible to improve extraction time and efficiency by adjusting voltages and geometry of the output electrodes. In the future it is planned to perform automatic search in order to find the optimal configuration.

\section{Conclusion}

Two different configurations for TESIS drift structure electrodes were considered. In the first one, ions drift to extraction area only under action of the electron string potential. In the second one, the device electrodes create azimuthal potential gradient, which speeds-up ion transition.

In both cases, it was possible to achieve about $70 \%$ ion extraction in $200 \mu \mathrm{s}$. The process is significantly faster in the configuration with azimuthal potential gradient: for $10 \mu$ s pulse beam intensity would be almost 6 times larger than without it. In further work, it is planned to improve extraction speed and efficiency by performing automatic search for the optimal configuration of electrodes geometry and potentials.

Authors would like to thank Dr. E.E. Donets and Dr. E.D. Donets for help and discussion. Software development for simulation was funded by RFBR according to the research project 18-32-00239. One of the authors (A.Yu.) would like to acknowledge support from JINR AYSS Grant. Computations were held on the basis of the heterogeneous computing cluster HybriLIT (LIT, JINR).

\section{References}

[1] E.D. Donets, Review of Scientific Instruments 67, 873 (1996)

[2] E.D. Donets, IEEE Transactions on Nuclear Science 23, 897 (1976)

[3] A. Boytsov, S. Gudkov, D.E. Donets, E.D. Donets, E.E. Donets, A. Ramsdorf, V. Salnikov, V. Shutov, RuPAC 2012 Contributions to the Proceedings pp. 208-212 (2012)

[4] E.D. Donets, D.E. Donets, E.E. Donets, Patent RU 2205467, 22.06 (2001) "Izobretenia" (2003)

[5] E.D. Donets, E.E. Donets, D.E. Donets, Review of Scientific Instruments 73, 696 (2002)

[6] E.D. Donets, E.E. Donets, Proceedings of EPAC-2002, Paris pp. 1703-1705 (2002)

[7] E.D. Donets et al., Review of Scientific Instruments 80, 063304 (2009)

[8] E.D. Donets et al., Proceedings of EPAC 2008 pp. 403-405 (2008)

[9] A. Boytsov, A. Bulychev, EPJ Web of Conferences 177 (2018)

[10] R.W. Hockney, J.W. Eastwood, Computer Simulation Using Particles (CRC Press, 1988) 